\title{
Exact Wave Packet Dynamics of Singlet Fission in Unsubstituted and Substituted Polyene Chains within Long-Range Interacting Models
}

\author{
Suryoday Prodhan ${ }^{1, *}$ and S. Ramasesha ${ }^{1, \oplus}$ \\ ${ }^{1}$ Solid State and Structural Chemistry Unit, Indian Institute of Science, Bangalore 560012, India
}

(Dated: September 27, 2018)

\begin{abstract}
Singlet fission (SF) is a potential pathway for significant enhancement of efficiency in organic solar cells (OSC). In this paper, we study singlet fission in a pair of polyene molecules in two different stacking arrangements employing exact many-body wave packet dynamics. In the noninteracting model, the SF yield is absent. The individual molecules are treated within Hubbard and Pariser-Parr-Pople (PPP) models and the interaction between them involves transfer terms, intersite electron repulsions and site-charge-bond-charge repulsion terms. Initial wave packet is constructed from excited singlet state of one molecule and ground state of the other. Time development of this wave packet under the influence of intermolecular interactions is followed within the Schrödinger picture by an efficient predictor-corrector scheme. In unsubstituted Hubbard and PPP chains, $2^{1} A$ excited singlet state leads to significant SF yield while the $1^{1} B$ state gives negligible fission yield. On substitution by donor-acceptor groups of moderate strength, the lowest excited state will have sufficient $2^{1} A$ character and hence results in significant SF yield. Because of rapid internal conversion, the nature of the lowest excited singlet will determine the SF contribution to OSC efficiency. Furthermore, we find the fission yield depends considerably on the stacking arrangement of the polyene molecules.
\end{abstract}

\section{INTRODUCTION}

Singlet fission (SF) is a process in which a molecule in the singlet excited state $\left(S_{n}\right)$ interacts with another molecule in the ground state $\left(S_{0}\right)$ resulting in triplet excited state on each molecule ${ }^{\underline{1}}$. Although this process can be described by a single step reversible pathway, a detailed scheme ${ }^{2}$ considering the hypotheses of spin-allowed transition is vividly accepted by the scientific community. In this scheme, interaction between the $S_{n}$ state and $S_{0}$ state results in a spin-singlet coupled ${ }^{1}\left(T_{1} T_{1}\right)$ state which later dissociates into two triplet excitons (Ref. 3-5 and references therein). Recently it has been reported that this multiexciton state is being observed experimentally via time-resolved two-photon photoemission spectroscopy ${ }^{6}$ and transient absorption and timeresolved photoluminescence spectroscopy $\underline{7}$. These studies also have shown more than $100 \%$ triplet yield from singlet excited state. In promising systems the rate constants for the fission of the singlet excited state should be higher, compared to other intra- and intermolecular processes like fluorescence. The energetics for singlet fission consists of two widely accepted requirements $3-$ (i) $E_{S_{n}} \geq 2 E_{T_{1}}$; systems with $E_{S_{n}}$ slightly less than $2 E_{T_{1}}$, have also been found to display signature of singlet fission due to vibronic processes (ii) the energy of the higher triplet state $\left(T_{2}\right)$ should be greater than $S_{n}$ energy to prevent intersystem crossing to the triplet state and also should be more than twice of $T_{1}$ energy to suppress refusion of the newly born triplets by triplet-triplet annihilation; however, in finite-size polyene chains $T_{2}$ remain

* Electronic mail: suryodayp@sscu.iisc.ernet.in

$\dagger$ Electronic mail: ramasesh@sscu.iisc.ernet.in lower compared to $S_{n}$, irrespective of the symmetry of the $S_{n}$ state and the second criteria is not met. In literature, $S_{1}$ which is the lowest singlet excited state is commonly considered to be the optically excited state. Michl and coworkers proposed a number of suitable SF candidates which satisfy the energetics criteria on the basis of single CI calculations within Pariser-Parr-Pople model ${ }^{8}$ and speculated that alternant hydrocarbons (notably polyacenes) and biradicaloids are good choices as chromophores for SF. In another study, Greyson and coworkers examined the appropriate strength of interchromophoric coupling necessary for singlet fission in some promising materials, employing density functional theory (DFT) $)^{9}$. Minami and Nakano gave a biradical description of singlet fission considering biradicaloid systems which have open-shell ground states 10 . They have also studied small-size oligorylenes $\frac{11}{}$ and alternant and non-alternant hydrocarbons 12 as singlet fission candidates employing the time-dependent density functional theory (TD-DFT). However, the notion that both lowest singlet excited state and lowest triplet state can be described by HOMO-LUMO excitations from the ground state is too crude for $\pi$-conjugated systems.

In this paper, we have gone beyond the static quantum chemical approach and studied the quantum dynamics of singlet fission. We have considered dimers of 1,3-butadiene, 1,3,5-hexatriene and 1,3,5,7-octatetraene in full configuration interaction space of the $\pi$-system within Hubbard and Pariser-Parr-Pople (PPP) model Hamiltonians. In the literature, there exist only a few studies which go beyond frontier molecular orbitals approximation $13-21$. The polyene systems are important model molecules and there are several reports which indicate singlet fission in carotenoids and polyene systems ${ }^{21-28}$. We start with a wave packet formed from the ground state of one molecule and the singlet excited 
state of another, these states being the exact eigenstates of the molecule within the chosen model Hamiltonians. We then introduce intermolecular interactions and evolve the wave packet in time. At each time step, the evolved wave packet is projected on to various direct products of the eigenstates of individual molecules in the triplet manifold to obtain the yield of the triplets. The time evolution is carried out in the full configuration space of the total $\pi$-system.

Effect of crystal stacking on SF efficiency have been studied extensively for acenes and other hydrocarbons ${ }^{29}-33$. In most of the materials, slipped stacked arrangement results in higher singlet fission yield as intermolecular vibrational modes which lead to direct coupling between the $S_{0} S_{1}$ state and ${ }^{1}\left(T_{1} T_{1}\right)$ state are sensitive to crystal packing. However, reports by Friend et al. and Guldi et al. on solution phase SF for substituted pentacene pointed out that SF is not confined to specific geometries and can be observed even in disordered systems 34,35 . Similar conclusion is also arrived at by Sanders and coworkers who studied SF of bipentacene in solution phase ${ }^{36}$.

The $S_{1}$ states in these polyenes are optically inactive and are primarily composed of two triplets 37.38 . Substitution in these moieties by donor-acceptor groups breaks the electron-hole and inversion symmetries making $S_{1}$ state optically active and therefore the lowest optical state in these systems shifts from $S_{2}$ to $S_{1}$. However, for weak symmetry breaking $S_{1}$ state continues to show the characteristics of two triplets. Thus we find that even in substituted polyenes, if the initial state is an $S_{1}$ state rather than other higher energy singlet state, $\mathrm{SF}$ is efficient. This agrees with some recent studies which suggest substitution by heteroatoms within organic chromophore $\frac{16,39,40}{4}$ or copolymerising donor-acceptor moieties $14-43$ can play an important role in tailoring candidate molecules for SF. The mixing of different eigenstates on donor-acceptor substitution, although well-known in $\pi$-conjugated carbon systems, its significance in singlet fission has not been explored. Our model study will be helpful in providing insights for developing better systems for SF.

In recent years, there is also considerable interest in intramolecular SF (iSF) in polymers. There are primarily two classes of systems which have been widely studied. In one class, the polymers consists of chromophores linked via conjugated linkers or covalent linkers which lead to through-bond or through-space interactions respectively. The widely studied systems have primarily polyacene chromophores like tetracene ${ }^{44-49}$, pentacene $\stackrel{18,35,36,50-57}{ }$, 1,3-diphenylisobenzofuran ${ }^{58}$ or terrylenediimide ${ }^{59}$. iSF studies have also been reported on bithiophene 60,61 and P3TV polymer ${ }^{27}$ which belong to the same class. The second class of systems consist of strong donor-acceptor units in the polymer which act as chromophores. Some notable candidates belonging to this class of iSF systems are PBTDO1 and PBTDO2 ${ }^{41}$, PDTP-DFBT ${ }^{42}$ and $\mathrm{PTB}^{43}$. In our study, chromophores in the respective systems are two polyene chains which have through-space interactions due to molecular stacking. Hence, our study can also be viewed as intramolecular SF with throughspace interactions between chromophores. Since, the difference between intramolecular and intermolecular SF is more semantic than substantial, a time evolution study of intramolecular SF will also proceed along similar lines as our study.

In the following section, we have given a brief account of the model Hamiltonians and methodology used in our study. In section III, we have discussed the pictures which emerge for unsubstituted polyenes within different model Hamiltonians along with the role of substitution in singlet fission yield for different alkene chain. In section IV, we summarize our study.

\section{METHODOLOGY}

In our study, the individual molecules considered are polyenes which have chain lengths, $N$ varying from 4 to 8 sites and are modelled by the Pariser-Parr-Pople (PPP) Hamiltonian ${ }^{62,63}$, which includes long-range electron correlations along with on-site Hubbard interaction $(U)$. The Hamiltonian of individual polyene is given by:

$$
\begin{gathered}
H_{\text {intra }}=\sum_{i=1}^{N-1} t_{0}\left(1-(-1)^{i} \delta\right)\left(\hat{E}_{i, i+1}+\text { H.C. }\right)+\sum_{i=1}^{N} \epsilon_{i} \hat{n}_{i} \\
+\sum_{i=1}^{N} \frac{U}{2} \hat{n}_{i}\left(\hat{n}_{i}-1\right)+\sum_{i>j=1}^{N} V_{i j}\left(\hat{n}_{i}-z_{i}\right)\left(\hat{n}_{j}-z_{j}\right) \\
\hat{E}_{i, i+1}=\sum_{\sigma} \hat{c}_{i, \sigma}^{\dagger} \hat{c}_{i+1, \sigma}
\end{gathered}
$$

where $t_{0}$ is the average transfer integral; $\delta$ is the strength of dimerization; $\epsilon_{i}$ is the site energy at the $i$-th site; $U$ is the Hubbard correlation strength and $V_{i j}$-s are the intersite electronic correlation strengths; $z_{i}$ is the local chemical potential at site $i$ which leaves the site neutral (for carbon in a $\pi$-conjugated system $\left.z_{i}=1\right)$. $\hat{c}_{i, \sigma}^{\dagger}\left(\hat{c}_{i, \sigma}\right)$ creates (annihilates) an electron of spin $\sigma$ in the orbital at $i$-th site and $\hat{n}_{i}$ is the corresponding number operator. Standard PPP parameters for carbon are employed, namely $t_{0}=-2.40 \mathrm{eV}$ and $U=11.26 \mathrm{eV} . \delta$ is taken as 0.07 and the $\mathrm{C}-\mathrm{C}$ bond lengths are fixed at $1.40\left(1+\frac{\delta}{2}\right)$ $\AA$ for the single bond and $1.40\left(1-\frac{\delta}{2}\right) \AA$ for the double bond. The long-range Coulomb interaction term $V_{i j}$ between site 'i' and ' $\mathrm{j}$ ' is parameterized using Ohno interpolation scheme $\frac{64}{}$,

$$
V_{i j}=14.397\left[\left(\frac{14.397}{U}\right)^{2}+r_{i j}^{2}\right]^{-\frac{1}{2}}
$$

which is arrived at by interpolating between $U$ at $r_{i j}=0$ and $e^{2} / r_{i j}$ for $r_{i j} \rightarrow \infty$. In Eq. 2, distance between site 
(A)
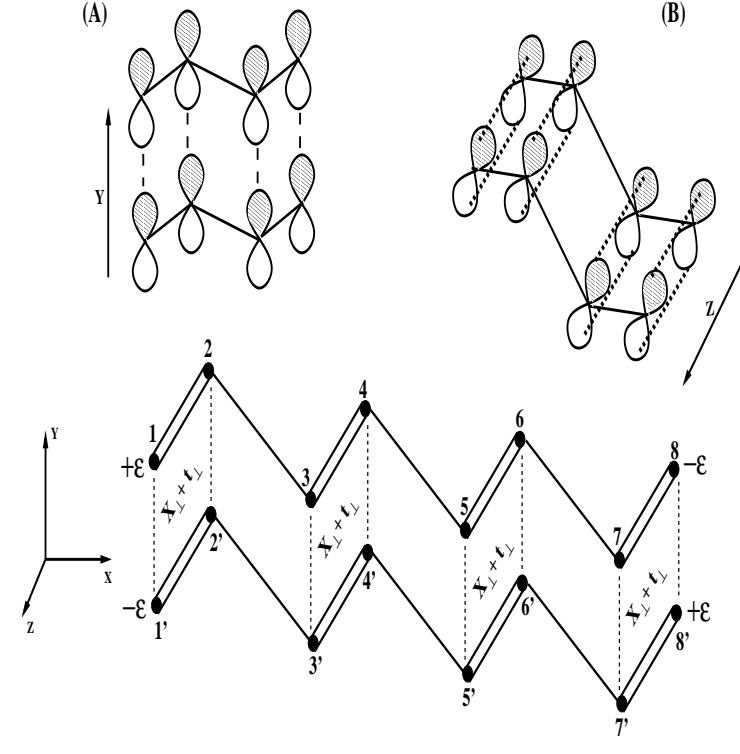

FIG. 1. Schematic diagram of the stacked polyenes; (A) in vertical stacking, monomers get stacked along Y-axis; (B) in horizontal stacking, monomers get stacked along Z-axis. $\mathrm{XY}$ plane is the molecular plane. The broken lines represent the intermolecular hopping interaction. $+\epsilon$ and $-\epsilon$ represent the donor and acceptor sites respectively while $\delta$ is the dimerization factor. The intrachain transfer integrals are taken to be $t_{0}(1 \pm \delta)$ for double/single bonds and corresponding bond lengths are taken to be $r_{0}(1 \mp \delta / 2)$. $t_{0}$ and $r_{0}$ are chosen to be $2.40 \mathrm{eV}$ and $1.4 \AA$ respectively. Site indices on different molecules are differentiated by using 'prime' superscript for sites on one molecule and without 'prime' for the other molecule.

'i' and ' $\mathrm{j}$ ' $\left(r_{i j}\right)$ is in $\AA$ while the energies are in $\mathrm{eV}$ 드. To study the role of substitution, site energies are varied at the chain ends to mimic donor and acceptor groups. Positive site energies correspond to donor groups and negative site energies to acceptor groups while site energies of unsubstituted carbon atoms are all set to zero. In our study, we have varied the strength of donor-acceptor substitution, $|\epsilon|$, from 0 to $5 \mathrm{eV}$.

If all long-range intersite interaction terms in the Hamiltonian are discarded, it represents Hubbard Hamiltonian. Singlet fission in unsubstituted systems are also studied within this model Hamiltonian, as a function of $U / t_{0}$ to probe the role of correlation strength. The $U / t_{0}=0$ case will reproduce the non-interacting or Hückel picture. In both Hückel and Hubbard models, we have considered $t_{0}=-1.0 \mathrm{eV}$ and the dimerization strength same as in the PPP model.

The above Hamiltonians, being non-relativistic conserves total spin $S_{\text {total }}$, along with z-component of total spin $\left(S_{z, \text { total }}\right)$. As we are primarily concerned with singlet and triplet manifolds, we work with valence bond (VB) basis which are eigenstates of total spin and employ the diagrammatic valence bond (DVB) method 66,67 for obtaining eigenstates in different spin subspaces for the monomers. Though complete and linearly independent, these basis states are non-orthogonal and result in nonsymmetric sparse Hamiltonian matrices for the polyenes in question. The Hamiltonians are fully diagonalized in each case to obtain the complete spectrum within the singlet and triplet subspaces of individual polyenes.

For probing singlet fission, we have considered two polyene monomers arranged in an eclipsed conformation with the separation between the two set at $4 \AA$. The stacking orientation of the two monomers can be either "vertical" (V stacking), where one monomer remains on top of another or "horizontal" (H stacking), as shown in Fig. 1(A) and 1(B). In both orientations, these monomers remain in an electrostatically favorable stacking configuration where the donor (acceptor) site of molecule I lies directly above the acceptor (donor) site of molecule II (Fig. 1).

The intermolecular Hamiltonian between the two monomers is given by:

$$
\begin{aligned}
H_{\text {inter }} & =\sum_{\left\langle i, i^{\prime}\right\rangle} t_{\perp}\left(\hat{E}_{i, i^{\prime}}+\hat{E}_{i^{\prime}, i}\right) \\
& +\sum_{i} \sum_{j^{\prime}} V_{i j^{\prime}}\left(\hat{n}_{i}-z_{i}\right)\left(\hat{n}_{j^{\prime}}-z_{j^{\prime}}\right) \\
& +\sum_{\left\langle i, i^{\prime}\right\rangle} X_{\perp}\left(2 \hat{n}_{i}+2 \hat{n}_{i^{\prime}}-2\right)\left(\hat{E}_{i, i^{\prime}}+\hat{E}_{i^{\prime}, i}\right)
\end{aligned}
$$

where $t_{\perp}$ is the inter-polyene hopping term between corresponding sites $i$ and $i^{\prime}$ on chains I and II which are directly above each other (Fig. 10). The transfer term is negative for horizontal stacking while it is positive for vertical stacking due to opposite signs of the overlap integrals (Fig. 1); in our calculations, we have considered $\left|t_{\perp}\right|=0.25 \mathrm{eV}$ in the PPP model and $0.2 \mathrm{eV}$ within Hückel and Hubbard models. The electron repulsion term comparable to the inter-molecular transfer term is $X_{\perp}$, the site-charge-bond-charge repulsion term and represents the two-electron integral $\left[i i \mid i i^{\prime}\right]$ and other related integrals within the charge cloud notation (Ref. 68); the other relevant multielectron repulsion term, bondcharge-bond-charge repulsion, represented by $\left[i i^{\prime} \mid i i^{\prime}\right]$, is neglected as it is expected to be much smaller compared to $X_{\perp}$ (Ref. 69). The site-charge-bond-charge term is neglected in the intra-molecular Hamiltonian as it affects only weakly the excitation spectrum of the isolated molecule. We have also taken $X_{\perp}=0$ for pair of sites on the two molecules which are not directly above each other. In charge cloud notation, the contribution to the Hamiltonian due to the repulsion term between site-charge at $i$ (of chain $\mathrm{I}$ ) and the bond between $i$ and $i^{\prime}$ (of chain II) can be denoted by the parameter $X_{\perp,\left\langle i i, i i^{\prime}\right\rangle}=\left[i i \mid i i^{\prime}\right]+\left[i i \mid i^{\prime} i\right]+\left[i i^{\prime} \mid i i\right]+\left[i^{\prime} i \mid i i\right]$; all integrals in this expression are equal and the corresponding second quantized operators are $\left(\hat{n}_{i}-1\right) \hat{E}_{i i^{\prime}}, \hat{n}_{i} \hat{E}_{i^{\prime} i}, \hat{n}_{i} \hat{E}_{i i^{\prime}}$ and $\left(\hat{n}_{i}-1\right) \hat{E}_{i^{\prime} i}$ respectively. Equivalent repulsion parameter $X_{\perp,\left\langle i^{\prime} i^{\prime}, i i^{\prime}\right\rangle}$ will also generate four interaction terms in the intermolecular Hamiltonian. In the non-interacting (Hückel) picture, $X_{\perp}$ term is taken to be zero. The pathways which lead to SF products from the $t_{\perp}$ and $X_{\perp}$ terms are schematically shown in Fig. 2 


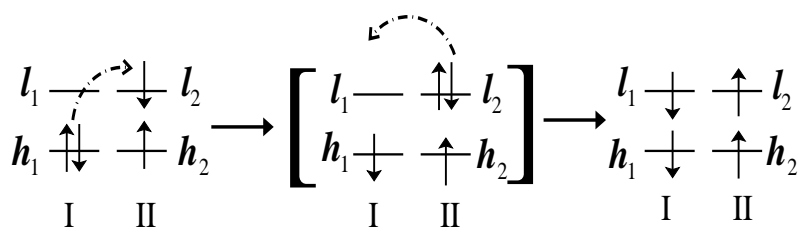

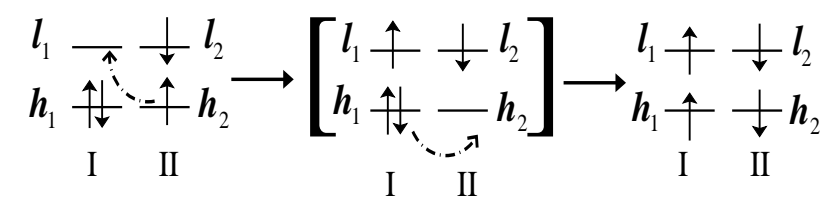

(A)

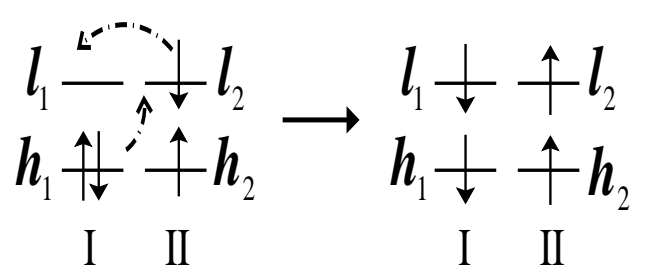

(B)

FIG. 2. (A) Schematic diagram of singlet fission pathway involving two inter-chromophoric one-electron hopping interactions $\left(t_{\perp}\right)$. $h_{1}$ and $l_{1}$ are the HOMO and LUMO of molecule 1 while $h_{2}$ and $l_{2}$ are those of molecule 2. The Hermitian conjugate of each step can be represented by reversing the direction of the broken arrow. (B) Schematic diagram of another pathway via two-electron repulsion integral $\left(X_{\perp}\right)$ involving frontier molecular orbitals. The two-electron operators are $\left[l_{1} l_{2} \mid l_{2} h_{1}\right]\left(\hat{E}_{l_{1} l_{2}} \hat{E}_{l_{2} h_{1}}-\hat{E}_{l_{1} h_{1}}\right),\left[l_{1} l_{2} \mid h_{1} l_{2}\right] \hat{E}_{l_{1} l_{2}} \hat{E}_{h_{1} l_{2}}$, $\left[l_{2} l_{1} \mid l_{2} h_{1}\right] \hat{E}_{l_{2} l_{1}} \hat{E}_{l_{2} h_{1}},\left[l_{2} l_{1} \mid h_{1} l_{2}\right] \hat{E}_{l_{2} l_{1}} \hat{E}_{h_{1} l_{2}}$ and their Hermitian conjugates, where $\hat{E}_{i j}$ represents $\sum_{\sigma} \hat{a}_{i, \sigma}^{\dagger} \hat{a}_{j, \sigma}$.

To justify our treatment, it is important to show that the intermolecular interactions are weak enough to describe the state of the full system approximately by a product of the eigenstates of the individual polyenes. To demonstrate this, we have computed several properties in the low-lying eigenstates of the full system and compared them with those of isolated polyenes (Table. I and Table. S1-S6 in the supplemental material $\left.{ }^{70}\right)$. These properties are (i) the projection of the low-lying eigenstates the full system onto the direct product of the low-lying eigenstates of isolated molecules (ii) average double occupancy of the sites in the monomer, and (iii) the spin of the monomer block in the full system calculated from the expectation value of the total spin $\left(S^{2}\right)$ for the block, using spin-spin correlation functions.

The ground state of the full system always has a very large projection on to the direct product of the ground states, namely, $S_{0} \otimes S_{0}$. The double occupancy in the fragments is also the same as in the isolated molecules. The total spin of the fragments is also nearly zero. In the excited states there is always a singlet in the covalent space which has large projection to both the $T_{1} \otimes T_{1}$ state and the $S_{n} \otimes S_{0}$ state. However, the average double occupancy of the sites from the two monomer states are very nearly the same as that of the full system. In this case, the spin expectation value on the fragments is large. In the case of other singlets in the ionic space, the projection on to the direct product of the isolated monomer states is very large. This analysis shows that the interaction term is a small perturbation on the isolated molecules.

Dynamics of a wave packet, which is direct product of a specified excited singlet state $\left|S_{n}^{I}\right\rangle$ of monomer I and the ground state $\left|S_{0}^{I I}\right\rangle$ of monomer II, is studied in the Schrödinger picture employing the full system Hamiltonian $H_{\text {full }}=H_{\text {intra }}+H_{\text {inter }}$. We have chosen the Schrödinger picture over the interaction picture as the full space of the dimer is too large to study within the interaction picture. It is convenient to obtain the eigenstates (both singlet and triplet) of the isolated molecules in the VB basis and convert them into Slater basis. The Hamiltonian matrix corresponding to $H_{\text {full }}$ is generated in the Hilbert space with $S_{z}^{f u l l}=0$ using Slater basis. The wavepacket is time evolved employing the fourthorder multistep differencing scheme (MSD4) ${ }^{71}$, given by:

$$
\begin{aligned}
& |\psi(t+2 \Delta t)\rangle=|\psi(t-2 \Delta t)\rangle+\frac{4 i H_{\text {full }} \Delta t}{3}[|\psi(t)\rangle \\
& -2(|\psi(t+\Delta t)\rangle+|\psi(t-\Delta t)\rangle)]+O\left(\left(H_{\text {full }} \Delta t\right)^{5}\right)
\end{aligned}
$$

as the predictor and the fourth-order Adams-Moultan scheme (Eq. 5) as the corrector.

$$
\begin{array}{r}
|\psi(t+2 \Delta t)\rangle=|\psi(t+\Delta t)\rangle-\frac{i H_{f u l l} \Delta t}{24}[9|\psi(t+2 \Delta t)\rangle \\
+19|\psi(t+\Delta t)\rangle-5|\psi(t)\rangle+|\psi(t-\Delta t)\rangle]
\end{array}
$$

This predictor-corrector scheme $\mathrm{T}^{72,73}$ is found to be very robust with accuracy comparable to the unconditionally stable Crank-Nicholson (CN) scheme ${ }^{74}$.

$$
\begin{aligned}
& \left(1+i H_{\text {full }} \Delta t / 2 \hbar\right)|\psi(t+\Delta t)\rangle \\
& \quad=\left(1-i H_{\text {full }} \Delta t / 2 \hbar\right)|\psi(t)\rangle+O\left(\left(H_{\text {full }} \Delta t\right)^{3}\right)
\end{aligned}
$$

The present time-evolution scheme is also less memory intensive and faster compared to the $\mathrm{CN}$ scheme; yet, the initial few steps of the evolution is carried out using the CN method. The validity of the above scheme is also examined by comparing the time evolution of small systems, calculated by exact methods like either evolving the initial state using the matrix representation of $\exp \left(-i H_{f u l l} \Delta t\right)$ or by projecting the initial state on the 
TABLE I. Properties of low-lying dimer eigenstates of unsubstituted butadiene and hexatriene dimers. $\langle\Psi \mid \Phi\rangle$ are projections of the dimer state on the direct product of the monomer states $|\Phi\rangle$, in the PPP model. The state $|\Phi\rangle$ is a simple direct product of the states when the states on the two monomers are the same. For two different monomer states, $|\phi\rangle$ and $|\chi\rangle,|\Phi\rangle=\frac{1}{\sqrt{2}}\left(|\phi\rangle_{I} \otimes|\chi\rangle_{I I}+|\chi\rangle_{I} \otimes|\phi\rangle_{I I}\right)$. $d_{I}$ and $d_{I I}$ are average double occupancy per site within monomer I and II while $s_{I}$ and $s_{I I}$ are the spin value of corresponding monomer units calculated from the spin-spin correlation functions. $d_{I / I I}^{(i s o)}$ are the average double occupancies of isolated monomer eigenstates. $\phi$-s and $\Psi$ are the eigenstates of the monomer and the dimer respectively.

\begin{tabular}{|c|c|c|c|c|c|c|c|c|c|c|c|}
\hline $\mathrm{N}$ & Orientation & $\Psi$ & $\phi_{I}$ & $\phi_{I I}$ & $|\langle\Psi \mid \Phi\rangle|$ & $d_{I}^{(i s o)}$ & $d_{I I}^{(i s o)}$ & $d_{I}$ & $d_{I I}$ & $s_{I}$ & $s_{I I}$ \\
\hline \multirow[t]{16}{*}{4} & \multirow[t]{8}{*}{$\mathrm{V}$} & $S_{0}$ & $S_{0}$ & $S_{0}$ & 0.99 & 0.17 & 0.17 & 0.17 & 0.17 & 0.004 & 0.004 \\
\hline & & \multirow[t]{2}{*}{$S_{1}$} & $S_{1}$ & $S_{0}$ & 0.44 & 0.10 & 0.17 & 0.13 & 0.13 & 0.81 & 0.81 \\
\hline & & & $T_{1}$ & $T_{1}$ & 0.83 & 0.11 & 0.11 & & & & \\
\hline & & $\overline{S_{2}}$ & $S_{2}$ & $S_{0}$ & 0.86 & 0.31 & 0.17 & 0.24 & 0.24 & 0.15 & 0.15 \\
\hline & & $S_{3}$ & $S_{1}$ & $S_{0}$ & 0.95 & 0.10 & 0.17 & 0.14 & 0.14 & 0.06 & 0.06 \\
\hline & & \multirow[t]{2}{*}{$\overline{S_{4}}$} & $S_{1}$ & $S_{0}$ & 0.88 & 0.10 & 0.17 & 0.13 & 0.13 & 0.33 & 0.33 \\
\hline & & & $T_{1}$ & $T_{1}$ & 0.46 & 0.11 & 0.11 & & & & \\
\hline & & $S_{5}$ & $S_{2}$ & $S_{0}$ & 0.96 & 0.31 & 0.17 & 0.24 & 0.24 & 0.05 & 0.05 \\
\hline & \multirow[t]{8}{*}{$\mathrm{H}$} & $S_{0}$ & $S_{0}$ & $S_{0}$ & 0.99 & 0.17 & 0.17 & 0.17 & 0.17 & 0.003 & 0.003 \\
\hline & & $\overline{S_{1}}$ & $S_{1}$ & $S_{0}$ & 0.35 & 0.10 & 0.17 & 0.12 & 0.12 & 0.90 & 0.90 \\
\hline & & & $T_{1}$ & $T_{1}$ & 0.92 & 0.11 & 0.11 & & & & \\
\hline & & $S_{2}$ & $S_{1}$ & $S_{0}$ & 0.99 & 0.10 & 0.17 & 0.14 & 0.14 & 0.01 & 0.01 \\
\hline & & $S_{3}$ & $S_{1}$ & $S_{0}$ & 0.93 & 0.10 & 0.17 & 0.14 & 0.14 & 0.22 & 0.22 \\
\hline & & & $T_{1}$ & $T_{1}$ & 0.36 & 0.11 & 0.11 & & & & \\
\hline & & $S_{4}$ & $S_{2}$ & $S_{0}$ & 0.98 & 0.31 & 0.17 & 0.24 & 0.24 & 0.04 & 0.04 \\
\hline & & $S_{5}$ & $S_{2}$ & $S_{0}$ & 0.98 & 0.31 & 0.17 & 0.24 & 0.24 & 0.04 & 0.04 \\
\hline \multirow[t]{16}{*}{6} & \multirow[t]{8}{*}{$\mathrm{V}$} & $S_{0}$ & $S_{0}$ & $S_{0}$ & 0.99 & 0.18 & 0.18 & 0.18 & 0.18 & 0.007 & 0.007 \\
\hline & & $S_{1}$ & $S_{1}$ & $S_{0}$ & 0.45 & 0.14 & 0.18 & 0.16 & 0.16 & 0.78 & 0.78 \\
\hline & & & $T_{1}$ & $T_{1}$ & 0.80 & 0.14 & 0.14 & & & & \\
\hline & & $S_{2}$ & $S_{1}$ & $S_{0}$ & 0.95 & 0.14 & 0.18 & 0.16 & 0.16 & 0.07 & 0.07 \\
\hline & & $S_{3}$ & $S_{2}$ & $S_{0}$ & 0.83 & 0.26 & 0.18 & 0.22 & 0.22 & 0.18 & 0.18 \\
\hline & & $S_{4}$ & $S_{1}$ & $S_{0}$ & 0.86 & 0.14 & 0.18 & 0.15 & 0.15 & 0.36 & 0.36 \\
\hline & & & $T_{1}$ & $T_{1}$ & 0.48 & 0.14 & 0.14 & & & & \\
\hline & & $\overline{S_{7}}$ & $S_{2}$ & $S_{0}$ & 0.95 & 0.26 & 0.18 & 0.22 & 0.22 & 0.06 & 0.06 \\
\hline & \multirow[t]{8}{*}{$\mathrm{H}$} & $S_{0}$ & $S_{0}$ & $S_{0}$ & 0.99 & 0.18 & 0.18 & 0.18 & 0.18 & 0.004 & 0.004 \\
\hline & & $\overline{S_{1}}$ & $S_{1}$ & $S_{0}$ & 0.41 & 0.14 & 0.18 & 0.15 & 0.15 & 0.87 & 0.87 \\
\hline & & & $T_{1}$ & $T_{1}$ & 0.90 & 0.14 & 0.14 & & & & \\
\hline & & $S_{2}$ & $S_{1}$ & $S_{0}$ & 0.99 & 0.14 & 0.18 & 0.16 & 0.16 & 0.01 & 0.01 \\
\hline & & $S_{3}$ & $S_{1}$ & $S_{0}$ & 0.90 & 0.14 & 0.18 & 0.16 & 0.16 & 0.28 & 0.28 \\
\hline & & & $T_{1}$ & $T_{1}$ & 0.42 & 0.14 & 0.14 & & & & \\
\hline & & $S_{4}$ & $S_{2}$ & $S_{0}$ & 0.96 & 0.26 & 0.18 & 0.22 & 0.22 & 0.06 & 0.06 \\
\hline & & $S_{5}$ & $S_{2}$ & $S_{0}$ & 0.96 & 0.26 & 0.18 & 0.22 & 0.22 & 0.05 & 0.05 \\
\hline
\end{tabular}

eigenstates of $H_{\text {full }}$ and explicitly evolving these eigenstates using their corresponding eigenvalues.

The Hamiltonian matrix used for the largest system in our study (16 carbon atoms) is of dimension $\sim 166$ million and for reasonable convergence, $\Delta t$ of the order of $0.002 \mathrm{eV} / \hbar$ is used for the PPP model, which is typically $\sim 0.00132 \mathrm{fs}$; for Hückel and Hubbard model, $\Delta t$ is taken as $0.01 \mathrm{eV} / \hbar(\sim 0.0066 \mathrm{fs})$. Hence, to follow the dynamics for just $30 \mathrm{fs}$, the time evolution has to be carried out for more than 20000 time steps within the PPP model and nearly 5000 time steps within the other two models.
After each time evolution step, the evolved state is projected onto the desired direct product of the triplet eigenstates of I and II i.e. $T_{m}^{I} \otimes T_{n}^{I I}$, where $T_{m}$ and $T_{n}$ are triplet eigenstates of individual monomers. The total $S_{z}$ value of the wave packet remains unaltered during time evolution, hence, the projection on the triplet channel is carried out in the same $S_{z}$ space; in this case, both monomers in the triplet state can have $S_{z}=0$ or one of them has $S_{z}=+1(-1)$ while the other has $S_{z}=-1(+1)$. Triplet eigenstates of individual monomers are calculated in $S_{z}=+1$ space using VB basis and employing $\hat{S}^{-}$operator, corresponding eigen- 
states in $S_{z}=0$ and -1 spaces are obtained. The yield in a given pair of triplet eigenstates $(m, n)$ is given by $I_{m, n}(t)=\left|\left\langle\psi(t) \mid T_{m}^{I} \otimes T_{n}^{I I}\right\rangle_{m, n}\right|^{2}$ where $\left|T_{m}^{I} \otimes T_{n}^{I I}\right\rangle=$ $\frac{1}{\sqrt{3}}\left|T_{m, S_{z}=0}^{I} \otimes T_{n, S_{z}=0}^{I I}\right\rangle-\frac{1}{\sqrt{3}}\left[\left|T_{m, S_{z}=+1}^{I} \otimes T_{n, S_{z}=-1}^{I I}\right\rangle+\right.$ $\left.\left|T_{m, S_{z}=-1}^{I} \otimes T_{n, S_{z}=+1}^{I I}\right\rangle\right]$, according to angular momentum algebra. However, the number of such pairs for a neutral subsystem can be enormous; number of triplet-triplet channels for octatetraene is 5,531,904 as each molecule has 2362 triplet states. Hence, the number of pairs to be investigated in each dimer system needs to be significantly reduced. This is achieved by restricting to $\sim 10$ low-lying triplet states on each of the neutral subsystems (corresponding to one hundred channels) and by applying a cut-off in the yield $\left(\sim 10^{-3}\right)$, which a channel must have at least at one step during the course of the full evolution.

\section{RESULTS AND DISCUSSION}

We have computed the integrated yield over the time period of evolution, defined as $I_{m, n}^{\text {total }}=\sum_{i} I_{m, n}\left(t_{i}\right) \Delta t$, where $I_{m, n}\left(t_{i}\right)$ is the yield at $i$-th step in triplet pair channel $(m, n)$ and $\Delta t$ is the time interval. Our model deals with static nuclei and hence vibronic or diabatic effects are ignored. We consider only the primary charge transfer process between two static molecules and the product associated with the process; the long-range interacting model is exactly solved with these caveats. The initial wave packet is not an eigenstate of the full Hamiltonian and hence evolves with time non-trivially under the influence of intermolecular interactions. During time evolution, the total energy of the wave packet is not conserved and the wave packet acquires non-zero components of the higher excited states through intermolecular interactions; however, the weights of these components are negligible. Therefore, we have ignored yields in these unphysical states. We have also observed Rabi type oscillations 75 expected from non-dissipative quantum dynamics. However, physically important final state is the $T_{1} \otimes T_{1}$ state and we focus only on this state in all our further discussions. As we have considered only $I_{1,1}(t)$, the subscript is dropped in all later discussions.

In our study, we have considered two different choices of the $S_{n}$ state. In the first, we have considered $S_{n} \equiv$ $S_{1}$; i.e., the lowest energy singlet excited state of one monomer and ground state of the other monomer is employed in constructing the initial wave packet. On the other hand, in the second, the lowest optical state is considered as the $S_{n}$ state $\left(S_{n} \equiv S_{o p}\right)$. Substitution by donor-acceptor groups at the end of the chains breaks spatial symmetry $\left(C_{2}\right)$ as well as electron-hole symmetry and results in mixing of eigenstates of different symmetries of the unsubstituted system; consequently, every eigenstate becomes optically allowed on substitution. In this case, we have considered the state with highest transition dipole moment from the ground state (within an energy window) as $S_{o p}$, i.e $S_{n} \equiv S_{o p}=S_{\mu_{t r}^{\max }}$. In all substituted polyenes, it has been assumed that the donor and acceptor strengths are same, i.e. $\left|\epsilon_{D}\right|=\left|\epsilon_{A}\right|=\epsilon$. For large enough $\epsilon, S_{1}$ and $S_{o p}$ states become same and there remains no difference between the two scenarios.

According to the Fermi's golden rule, the rate of transition probability from the initial state $|i\rangle$ to the final state $|f\rangle$ is given by:

$$
W_{i \rightarrow f}=\frac{2 \pi}{\hbar}\left|\left\langle i\left|H_{\text {inter }}\right| f\right\rangle\right|^{2} \rho_{f}\left(E_{f}\right)
$$

In the case of polyenes the density of excitonic states is given by $\delta\left(E-E_{f}\right)$ as the spectrum is discrete. This implies that the transition rate is completely governed by the matrix element $\left\langle i\left|H_{\text {inter }}\right| f\right\rangle$. We have computed the matrix element in both non-interacting and interacting models and found it to be negligible. Thus, within this simple approach, we will not observe any SF.

To study the SF process in detail and obtain physical insights, we have analyzed small polyene systems (dimer of unsubstituted or substituted 1,3-butadiene). We express the initial wave packet $\Psi(0)$ as a linear combination of the eigenstates $\psi_{k}$ of the full Hamiltonian with eigenvalues $E_{k}$. The time evolution of the wavepacket is carried out using the eigenvalues of the corresponding states, i.e., $\Psi(t)=\sum_{k} c_{k}\left|\psi_{k}\right\rangle \exp \left(-i E_{k} t / \hbar\right)$. The yield $I(t)$ in this approach is given by:

$$
\begin{gathered}
I(t)=\sum_{i}\left|\left\langle S_{n} \otimes S_{0} \mid \psi_{i}(0)\right\rangle\left\langle T_{1} \otimes T_{1} \mid \psi_{i}(0)\right\rangle\right|^{2} \\
+2 \sum_{i} \sum_{j>i} \operatorname{Re}\left\{\left\langle S_{n} \otimes S_{0} \mid \psi_{i}(0)\right\rangle\left\langle T_{1} \otimes T_{1} \mid \psi_{i}(0)\right\rangle\right. \\
\left.\left\langle\psi_{j}(0) \mid S_{n} \otimes S_{0}\right\rangle\left\langle\psi_{j}(0) \mid T_{1} \otimes T_{1}\right\rangle\right\} \cos \left(\omega_{i j} t\right) \\
\omega_{i j} \equiv\left(E_{i}-E_{j}\right) / \hbar
\end{gathered}
$$

From Eq. 8, it can be noted that for a high cross-section in singlet fission, it is necessary that at least one of the eigenstates of the full system Hamiltonian should have simultaneously large non-zero overlaps with the initial and final states.

In the Hückel model, the lowest energy excited state is also the optical state $\left(S_{1} \equiv 1^{1} B\right)$. The two-photon state remains much higher in energy compared to the optical state and we do not consider evolution from this state. The energies of the lowest optical state and the lowest triplet state are same in the Hückel picture. Thus energetically a single optically excited molecule cannot yield two triplets in the Hückel model. Indeed, yields in the $T_{1} \otimes T_{1}$ channel are zero for both $\mathrm{V}$ and $\mathrm{H}$ stackings (Fig. 1). The overlap integrals of the full system eigenstates with the initial and final states are not large simultaneously (Fig. $S 1$ in the supplemental material ${ }^{70}$ ), which is a prerequisite for large yield. Hence, without electron correlations, the cross-section for singlet fission will be negligible. 
V Stacking
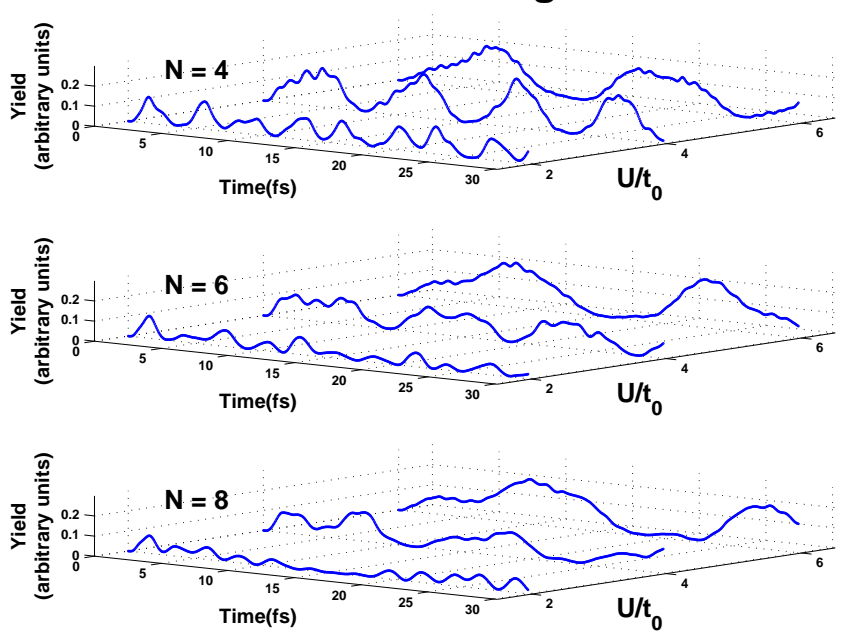

(A)
H Stacking
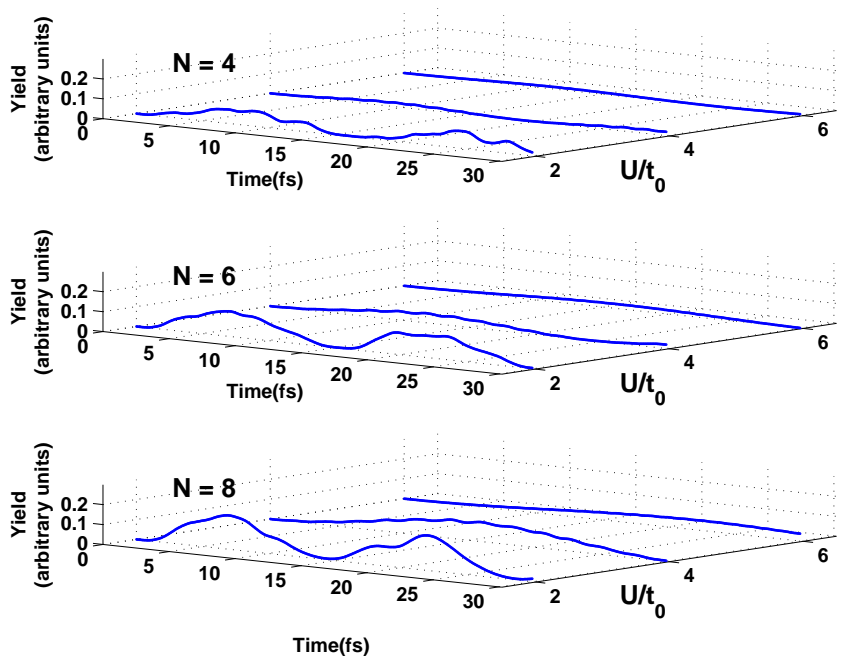

(B)

FIG. 3. (Color online) Time evolution profiles of different polyene dimers in V stacking $\left(t_{\perp}>0\right)(\mathrm{A})$ and $\mathrm{H}$ stacking $\left(t_{\perp}<0\right)(\mathrm{B})$ for different correlation strengths $\left(U / t_{0}\right)$; here, $S_{n} \equiv 2^{1} A$ case is considered.
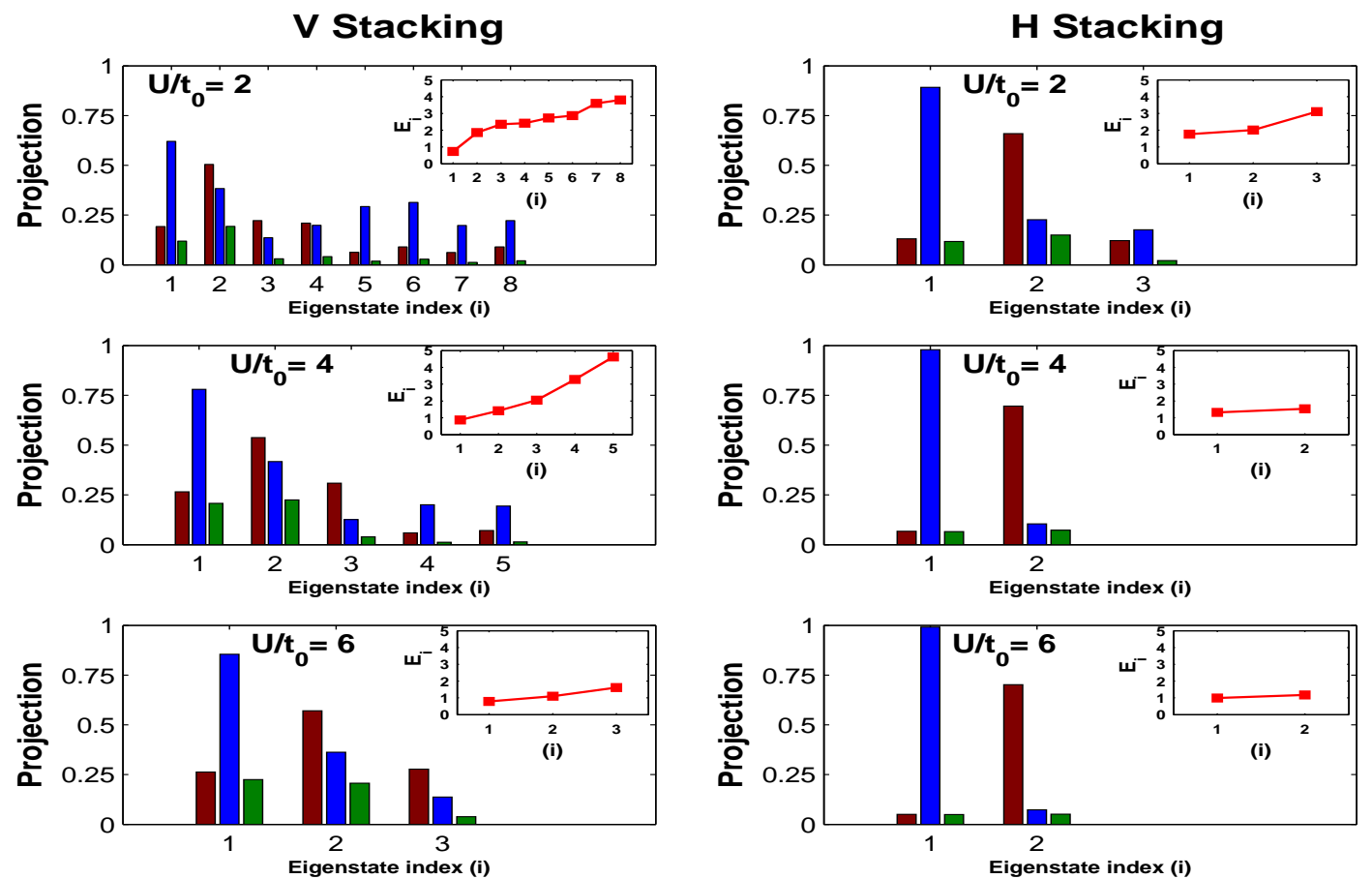

FIG. 4. (Color online) Significant projections of $2^{1} A \otimes 1^{1} A$ and $T_{1} \otimes T_{1}$ with full system eigenstates within Hubbard model are shown as histograms. The left panel corresponds to $\mathrm{V}$ stacking while the right panel corresponds to $\mathrm{H}$ stacking. The color indices are as follows: dark brown, projection to initial state, $P_{i} \equiv$ $\left\langle 2^{1} A \otimes 1^{1} A \mid \psi_{i}\right\rangle$; dark blue, projection to final state, $P_{f} \equiv\left\langle T_{1} \otimes T_{1} \mid \psi_{i}\right\rangle$; dark green, $P_{i} \times P_{f}$. Inset: $E_{i}$, the energy of the significant eigenstate ' $i$ ' as measured from the ground state of the full system is shown. 
TABLE II. Dependence of total yield $\left(I^{\text {total }}\right)$ on the parameters of $H_{\text {inter }}$ in the Hubbard and PPP model for a pair of butadiene and hexatriene and octatetraene. $X_{\perp}$ is the site-charge-bond-charge repulsion term which is either zero or $0.2 \mathrm{eV}$ for Hubbard model and $0.25 \mathrm{eV}$ for PPP model. $t_{\perp}$ is the intermolecular transfer term between corresponding sites and within Hubbard model in units of $t_{0}$, $t_{\perp}=+0.2$ in $\mathrm{V}$ stacking and $t_{\perp}=-0.2$ in $\mathrm{H}$ stacking. In the PPP model, we have taken $t_{\perp}=0.25 \mathrm{eV}$ in $\mathrm{V}$ stacking and $t_{\perp}=-0.25 \mathrm{eV}$ in $\mathrm{H}$ stacking.

\begin{tabular}{|c|c|c|c|c|c|c|c|c|}
\hline \multirow[t]{2}{*}{ System } & \multirow[t]{2}{*}{ Model } & \multirow[t]{2}{*}{$U / t_{0}$} & \multicolumn{2}{|c|}{$X_{\perp}=0, t_{\perp} \neq 0$} & \multicolumn{2}{|c|}{$X_{\perp} \neq 0, t_{\perp}=0$} & \multicolumn{2}{|c|}{$X_{\perp} \neq 0, t_{\perp} \neq 0$} \\
\hline & & & $\mathrm{V}$ & $\mathrm{H}$ & $\mathrm{V}$ & $\mathrm{H}$ & $\mathrm{V}$ & $\mathrm{H}$ \\
\hline \multirow{4}{*}{ butadiene } & \multirow{3}{*}{ Hubbard } & 2.0 & 1.70 & 1.70 & 2.32 & 2.32 & 1.62 & 1.18 \\
\hline & & 4.0 & 0.66 & 0.66 & 2.45 & 2.45 & 2.90 & 0.30 \\
\hline & & 6.0 & 0.26 & 0.26 & 1.84 & 1.84 & 2.65 & 0.13 \\
\hline & $\mathrm{PPP}$ & & 4.72 & 4.72 & 4.77 & 4.77 & 4.42 & 2.71 \\
\hline \multirow{4}{*}{ hexatriene } & \multirow{3}{*}{ Hubbard } & 2.0 & 2.47 & 2.47 & 2.01 & 2.01 & 0.86 & 1.91 \\
\hline & & 4.0 & 1.11 & 1.11 & 2.93 & 2.93 & 2.12 & 0.59 \\
\hline & & 6.0 & 0.64 & 0.64 & 2.47 & 2.47 & 3.21 & 0.35 \\
\hline & $\mathrm{PPP}$ & & 5.89 & 5.85 & 4.59 & 4.61 & 4.67 & 4.18 \\
\hline \multirow{4}{*}{ octatetraene } & \multirow{3}{*}{ Hubbard } & 2.0 & 2.98 & 2.98 & 1.32 & 1.32 & 0.80 & 2.34 \\
\hline & & 4.0 & 2.13 & 2.13 & 3.18 & 3.18 & 1.84 & 1.47 \\
\hline & & 6.0 & 1.57 & 1.57 & 3.10 & 3.10 & 3.16 & 0.89 \\
\hline & PPP & & 8.19 & 8.23 & 5.56 & 5.57 & 4.90 & 6.03 \\
\hline
\end{tabular}

We have studied singlet fission for a pair of butadienes, hexatrienes and octatetraenes within the Hubbard model for different on-site correlation strengths, $U / t_{0}$. In order to understand the role of $X_{\perp}$ term vis a vis that of $t_{\perp}$, we have studied three cases (i) $X_{\perp}=0 ; t_{\perp} \neq 0$, (ii) $X_{\perp} \neq 0 ; t_{\perp}=0$ and (iii) $X_{\perp} \neq 0 ; t_{\perp} \neq 0$ with $2^{1} A$ state as the initial singlet excited state (Table. III). In cases (i) and (ii), we find that the yield does not depend upon the type of stacking. However, the $X_{\perp}$ term gives rise to higher $\mathrm{SF}$ yield compared to $t_{\perp}$ term at larger $U / t_{0}$ values. In case (iii), when both $X_{\perp}$ and $t_{\perp}$ are nonzero, we find a synergistic effect on the SF yield in both stacking orientations. In the $\mathrm{V}$ stacking, the SF yield increases with correlation strength while in the $\mathrm{H}$ stacking it decreases with correlation strength. In the case of PPP model, we find that the yields are significantly larger than in the Hubbard model. Furthermore, the yield increases with chain length, showing the importance of intermolecular interactions. In the case of hexatriene and octatetraene dimers, $X_{\perp}$ term leads to a decrease in the yield in all cases. When both $X_{\perp}$ and $t_{\perp}$ are present, the yield is marginally higher for $\mathrm{H}$ stacking than in $\mathrm{V}$ stacking for longer oligomer.

To understand this behavior, we have focused on the bond order $\left(-\left\langle E_{i i^{\prime}}+E_{i^{\prime} i}\right\rangle / 2\right)$ between corresponding sites of the two molecules. The bond order is larger when $t_{\perp}<0$ and smaller when $t_{\perp}>0$. The larger bond order implies the site-charge densities are more uniform in the eigenstates of the full system. This leads to smaller contribution from the $X_{\perp}$ term in $\mathrm{H}$ stacking since the amplitude for hopping due to $X_{\perp}$ term is site-charge dependent.

When $S_{n} \equiv 1^{1} B$, total yields are insignificant in both $\mathrm{V}$ and $\mathrm{H}$ stackings. Analysis employing full system
Hamiltonian eigenstates shows highly disjoint overlaps with the initial and final states (Fig. S2 in the supplemental material $\left.{ }^{70}\right)$, similar to the Hückel model. Hence, only choice of $2^{1} A$ for the initial excited singlet state results in significant $I^{\text {total }}$ in both stackings (Fig. 3 and 5).

The time evolution profiles, shown in Fig. 3(A) and [3(B) shed light on the dependence of $I^{\text {total }}$ on monomer chain length in the Hubbard model. In $\mathrm{V}$ stacking, at a particular $U / t_{0}$, temporal variation of $I(t)$ in the evolution profile becomes weaker for longer chain systems, and the oscillatory pattern becomes more complex. In these cases, the eigenspectrum of dimers become more dense with increasing chain length and larger number of eigenstates contribute significantly towards $I(t)$ resulting in complex interference in the time evolution profile. The time evolution profiles (Fig. 3(B)) also suggest that the significant eigenstates in $\mathrm{H}$ stacking are almost degenerate as the yield shows simpler time dependence. This can also be seen from the right panel in Fig. 4.

Organic systems that we are interested in are semiconducting. Hence long-range interactions are not screened out as in metals and for a realistic modeling of the system we need to include explicit long-range electron-electron interactions. The PPP model with standard parameters is well suited for modeling conjugated organics $38,66,76-78$. We have found that introducing long-range interaction dramatically changes the yield of triplets. When the wave packet is built from an optical state $\left(1^{1} B\right)$ on one molecule and ground state on another, the total yield remains quite low in both $\mathrm{V}$ and $\mathrm{H}$ stackings. On the other hand, when the initial wave packet is constructed from $2^{1} A$ state and the ground state, there is significant increase in $I^{\text {total }}$, as can be seen from Figs. 5] and 6. For 
TABLE III. Full system eigenstates of 1,3-butadiene dimer having significant projections with $S_{n} \otimes 1^{1} A\left(P_{i}\right)$ and $T_{1} \otimes T_{1}\left(P_{f}\right)$ in the PPP model are tabulated for $\mathrm{V}$ and $\mathrm{H}$ stacking. $E$ is the excitation energy of the full system in $\mathrm{eV}$.

\begin{tabular}{|c|c|c|c|c|c|c|c|c|c|c|c|c|c|c|c|}
\hline \multicolumn{8}{|c|}{ V stacking } & \multicolumn{8}{|c|}{ H stacking } \\
\hline \multicolumn{4}{|c|}{$S_{n} \equiv 2^{1} A$} & \multicolumn{4}{|c|}{$S_{n} \equiv 1^{1} B$} & \multicolumn{4}{|c|}{$S_{n} \equiv 2^{1} A$} & \multicolumn{4}{|c|}{$S_{n} \equiv 1^{1} B$} \\
\hline$E$ & $P_{i}$ & $P_{f}$ & $P_{i} \times P_{f}$ & $E$ & $P_{i}$ & $P_{f}$ & $P_{i} \times P_{f}$ & $E$ & $P_{i}$ & $P_{f}$ & $P_{i} \times P_{f}$ & $E$ & $P_{i}$ & $P_{f}$ & $P_{i} \times P_{f}$ \\
\hline 4.71 & 0.3 & 0.8 & 0.2 & 4.71 & 0.0 & 0.8 & 0.0 & 5.28 & 0.2 & 0.9 & 0.2 & 5.28 & 0.0 & 0.9 & 0.0 \\
\hline \multirow[t]{7}{*}{5.32} & 0.6 & 0.5 & 0.3 & 4.93 & 0.6 & 0.0 & 0.0 & 5.34 & 0.6 & 0.4 & 0.2 & 5.34 & 0.0 & 0.4 & 0.0 \\
\hline & & & & 5.31 & 0.0 & 0.5 & 0.0 & & & & & 5.56 & 0.7 & 0.0 & 0.0 \\
\hline & & & & 5.84 & 0.7 & 0.0 & 0.0 & & & & & 5.90 & 0.7 & 0.0 & 0.0 \\
\hline & & & & 7.55 & 0.2 & 0.0 & 0.0 & & & & & & & & \\
\hline & & & & 8.18 & 0.3 & 0.0 & 0.0 & & & & & & & & \\
\hline & & & & 8.73 & 0.0 & 0.2 & 0.0 & & & & & & & & \\
\hline & & & & 11.04 & 0.0 & 0.1 & 0.0 & & & & & & & & \\
\hline
\end{tabular}

polyenes within the PPP model, large number of eigenstates have significant simultaneous projections on both $\left|2^{1} A \otimes 1^{1} A\right\rangle$ and $\left|T_{1} \otimes T_{1}\right\rangle$ (Table. 【II); these states are also nearly isoenergetic in $\mathrm{H}$ stacking leading to constructive interference (Eq. 8) and large yields (Fig. 7 and Figs. S3 and $\mathrm{S} 4$ in the supplemental material70). In V stacking, on the other hand, the contributing states have different energies and the yield is lower. It should be noted that this conclusion excludes effects of molecular vibrations or phonons on the SF process.

The total yield for varying $\epsilon$ is plotted in Fig. 6 while the time evolution profiles for octatetraene dimers for different substitution strength are shown in Fig. 73 corresponding time evolution profiles for butadiene and hexatriene are given in Figs. S3 and S4 in the supplemental material ${ }^{70}$. In $\mathrm{V}$ stacking, $I^{\text {total }}$ decreases with increasing substitution strength when $S_{n} \equiv S_{1}$ and this outcome is independent of monomer size (except for octatetraene dimer with $\epsilon=3.0)$. Yet, when $S_{o p}$, the state to which the transition dipole moment is largest is considered, non-monotonous behavior of $I^{\text {total }}$ is observed with increasing $\epsilon$. The total yield is dependent on the nature of the singlet excited state at small $\epsilon$. At large $\epsilon$
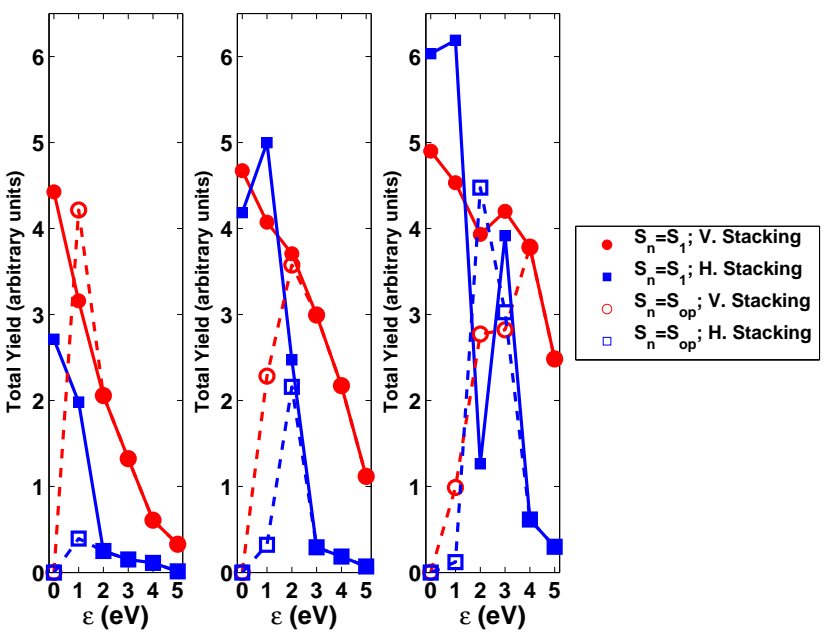

FIG. 6. (Color online) Total yield in unsubstituted and substituted polyene dimers are plotted as a function of substitution strength $\epsilon$ for both vertical and horizontal stacking orientations within the PPP model. The left, center and right panels correspond to monomers of 4,6 and 8 sites. The color and symbol indices are given in the following and same in all three panels: red filled circle, $S_{n} \equiv S_{1}$, V stacking; blue filled square, $S_{n} \equiv S_{1}, \mathrm{H}$ stacking. The open symbols represent $S_{n} \equiv S_{o p}$ scenarios in the corresponding systems. Beyond a certain $\epsilon, S_{n} \equiv S_{1} \equiv S_{o p}$ and the curves coincide. The solid and broken lines are given only as a guide to the eyes.
FIG. 5. (Color online) $I^{\text {total }}$ is plotted as a function of correlation strength $U / t_{0}$ for $S_{n} \equiv 2^{1} A$. The left, center and right panels correspond to monomers of 4,6 and 8 sites. Red filled circle corresponds to $\mathrm{V}$ stacking and blue filled square corresponds to $\mathrm{H}$ stacking. The broken lines in each panel correspond to the PPP values for unsubstituted system in $\mathrm{V}$ (red) and $\mathrm{H}$ (blue) stackings. The solid lines are given only as a guide to the eye. 


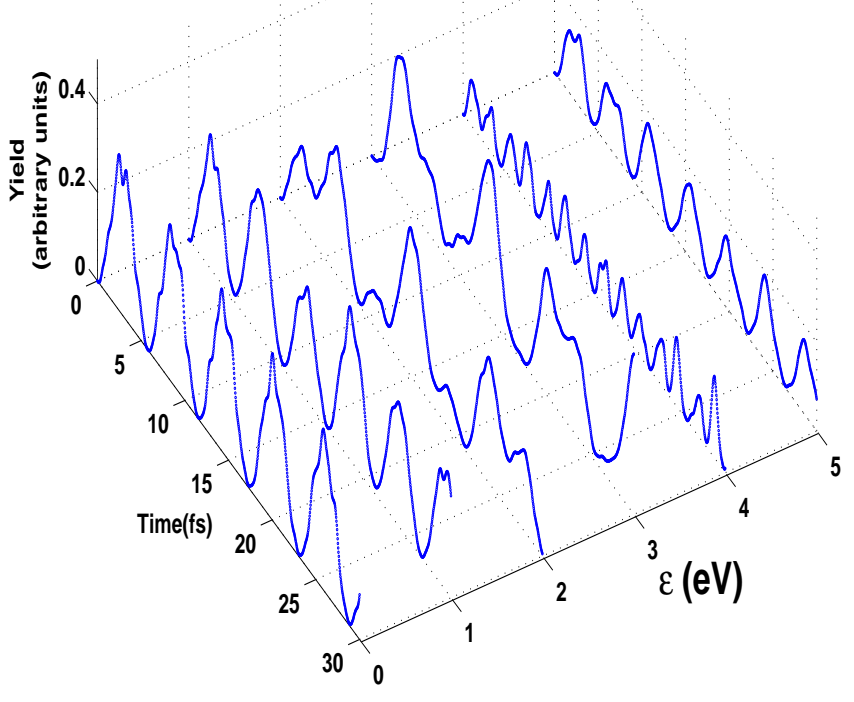

(A)

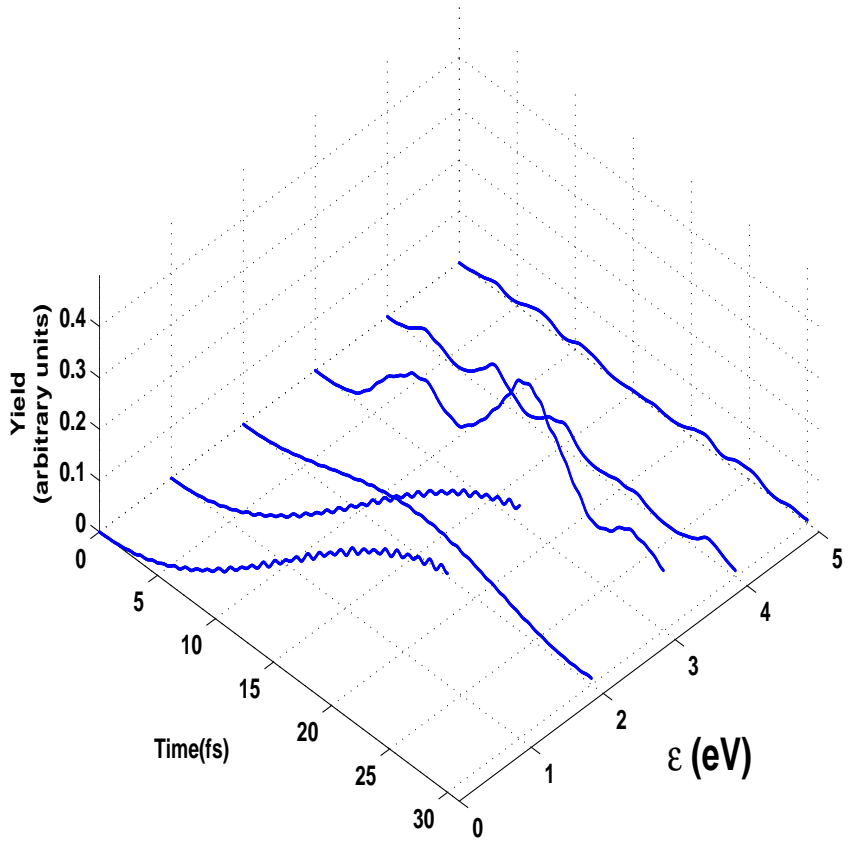

(B)

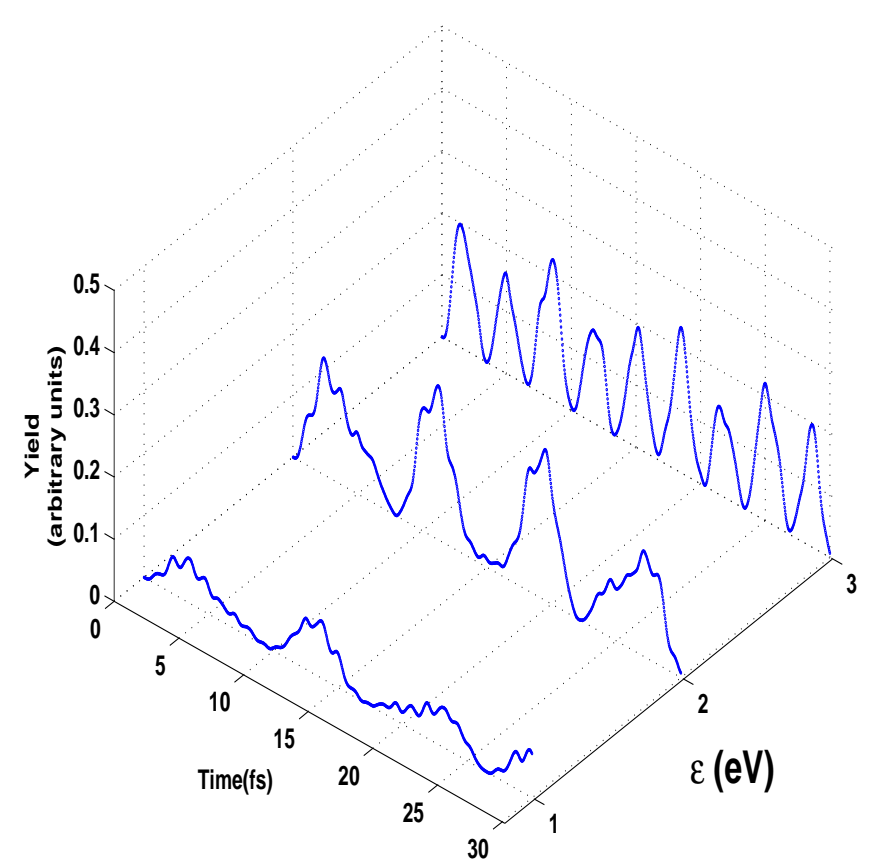

(C)

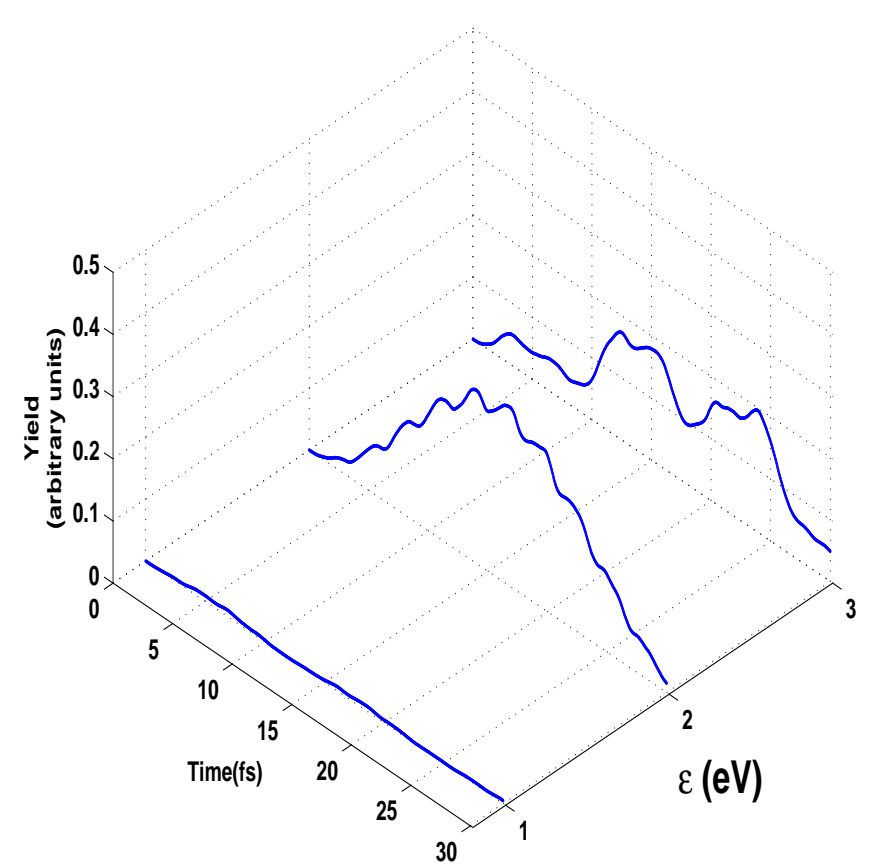

(D)

FIG. 7. (Color online) Yield as a function of time and donor-acceptor strength $\epsilon$ for singlet fission in 1,3,5,7-octatetraene dimer from the lowest singlet excited state $S_{1}$ in (A) V stacking and (B) $\mathrm{H}$ stacking. For $\epsilon=1 \mathrm{eV}, 2 \mathrm{eV}$ and $3 \mathrm{eV}$, the yield from the optical singlet state is also shown in (C) $\mathrm{V}$ stacking and (D) $\mathrm{H}$ stacking. For $\epsilon \geq 4 \mathrm{eV}$, we find that the lowest excited state is also the state with large transition dipole moment. 
TABLE IV. Energy gaps in butadiene, hexatriene and octatetraene within Hückel, Hubbard and PPP models. Energy gaps are given in units of $t_{0}$ within Hückel and Hubbard models.

\begin{tabular}{|c|c|c|c|c|c|c|}
\hline & & $U / t_{0}=0$ & $U / t_{0}=2$ & $U / t_{0}=4$ & $U / t_{0}=6$ & $\mathrm{PPP}(\mathrm{eV})$ \\
\hline \multirow{5}{*}{ butadiene } & $E_{1^{1} B}$ & 1.40 & 2.19 & 3.40 & 4.94 & 5.83 \\
\hline & $E_{2^{1} A}$ & 2.33 & 2.06 & 1.54 & 1.17 & 5.34 \\
\hline & $E_{T_{1}}$ & 1.40 & 0.96 & 0.67 & 0.50 & 2.67 \\
\hline & $E_{1^{1} B}-2 E_{T_{1}}$ & -1.40 & 0.27 & 2.06 & 3.94 & 0.49 \\
\hline & $E_{2^{1} A}-2 E_{T_{1}}$ & -0.47 & 0.14 & 0.20 & 0.17 & 0.00 \\
\hline \multirow{5}{*}{ hexatriene } & $E_{1^{1} B}$ & 1.07 & 1.68 & 2.78 & 4.26 & 5.05 \\
\hline & $E_{2^{1} A}$ & 1.81 & 1.63 & 1.25 & 0.96 & 4.36 \\
\hline & $E_{T_{1}}$ & 1.07 & 0.76 & 0.56 & 0.42 & 2.18 \\
\hline & $E_{1^{1} B}-2 E_{T_{1}}$ & -1.07 & 0.16 & 1.66 & 3.42 & 0.69 \\
\hline & $E_{2^{1} A}-2 E_{T_{1}}$ & -0.33 & 0.11 & 0.13 & 0.12 & 0.00 \\
\hline \multirow{5}{*}{ octatetraene } & $E_{1^{1} B}$ & 0.88 & 1.39 & 2.44 & 3.90 & 4.56 \\
\hline & $E_{2^{1} A}$ & 1.47 & 1.37 & 1.07 & 0.83 & 3.75 \\
\hline & $E_{T_{1}}$ & 0.88 & 0.66 & 0.49 & 0.38 & 1.90 \\
\hline & $E_{1^{1} B}-2 E_{T_{1}}$ & -0.88 & 0.07 & 1.46 & 3.14 & 0.76 \\
\hline & $E_{2^{1} A}-2 E_{T_{1}}$ & -0.29 & 0.05 & 0.09 & 0.07 & -0.05 \\
\hline
\end{tabular}

the lowest excited singlet state is also the most strongly optically allowed singlet state and the distinction ceases. In contrast, $\mathrm{H}$ stacking orientation exhibits unique $I^{\text {total }}$ profile with increasing $\epsilon$ (Fig. 6); large variation in $I^{\text {total }}$ as a function of $\epsilon$ is observed for both $S_{n} \equiv S_{1}$ and $S_{n} \equiv S_{o p}$ cases. This general trend is observable in all three polyene systems considered.

The energetics show that the singlet fission process is either slightly exoergic or endoergic for substituted PPP chains (Fig. 8). From energy consideration, we note that donor-acceptor strength $\epsilon$ between 2.0 and $3.0 \mathrm{eV}$ would result in large SF yield.
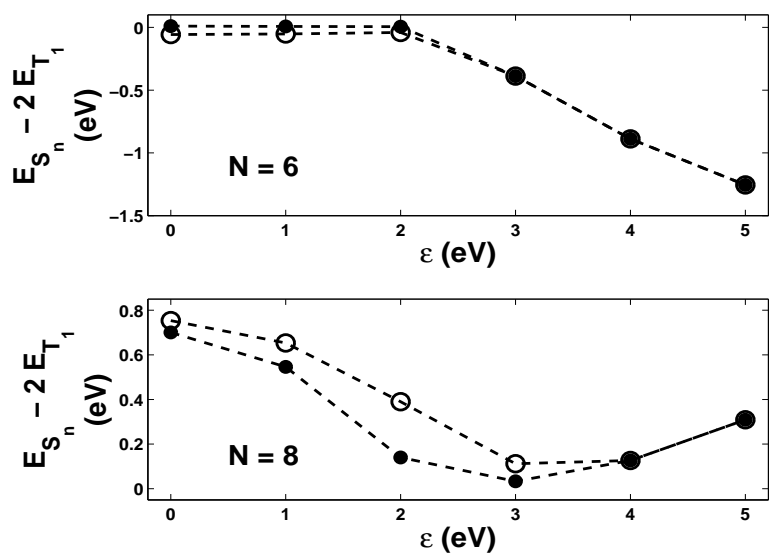

FIG. 8. Energy difference between the initial and final coupled states $\left(E_{S_{n}}-2 E_{T_{1}}\right)$ within PPP model are plotted against various $\epsilon$ for hexatriene and octatetraene. Filled circles correspond to $S_{N} \equiv S_{1}$ while open circles correspond to $S_{n} \equiv S_{o p}$ scenario. For $\epsilon=0, S_{1} \equiv 2^{1} A$ while $S_{o p}$ is the lowest energy state of $B$ symmetry subspace $\left(S_{o p} \equiv 1^{1} B\right)$. In substituted PPP dimers, beyond a particular $\epsilon, S_{1}$ becomes equivalent with $S_{o p}$ and the curves coincide. The broken lines are shown only as a guide to the eyes.

\section{CONCLUSION}

The energy criteria proposed by Michl et al. (Ref. 3 5) that singlet fission is feasible when the initial state energy is greater than or equal to the final state energy is seen to be operative in our model studies. In the Hückel model the optical $1^{1} B$ state is degenerate with the triplet state and as a consequence the energy criteria is not met, resulting in insignificant singlet fission yield. The energy of the $2^{1} A$ state in the Hückel model is also not close to the total energy of two triplets and hence $2^{1} A$ will also not yield singlet fission products in non-interacting models.

When electron correlations are turned on, as in the Hubbard or PPP models, the energy of the triplet state, being covalent, comes down while the energy of $1^{1} B$ state, being ionic, increases. There is a crossover in the $1^{1} B$ and $2^{1} A$ states, depending upon the correlation strength and chain length 77,79 . The energy of the $2^{1} A$ state is nearly twice the energy of lowest triplet state (Table. IV] and hence, an initial singlet excitation in $2^{1} A$ state yields significant triplets in the singlet fission process. However, the $1^{1} B$ state is not energetically close to two triplets and yields insignificant SF products. Analysis on butadiene dimer shows that in all models, the simultaneous overlap of the wave packet and the final product state with the eigenstates of $H_{\text {full }}$ is negligible when the wave packet is formed from $1^{1} B$ state. On the other hand, for wave packet constructed from $2^{1} A$ state, the simultaneous overlap is significant for some eigenstates, thereby leading to fission products. Indeed, we also find from the analysis of the full system eigenstates that there is a singlet excited state of the full system which is a coherent state of the $2^{1} A$ singlet and two triplets. Since 
in reality, the excitations occur in the full system, we can conclude that the primary excitation is to an optically allowed excitation, which leads to this coherent state through internal conversion. Our studies also show that excitation to $1^{1} B$ state does not directly yield SF products as suggested by Musser et al, 27,28. This is also because the $1^{1} B$ excitation is to an ionic state while the $2^{1} A$ and the triplets involved in SF are covalent states. Our studies are also in agreement with earlier PPP studies which postulate a coherent state $\frac{42}{2}$ as well as those that show the importance of the $2^{1} A$ state ${ }^{80}$.

In substituted polyene chains, for small donor-acceptor strengths singlet state derived from $1^{1} B$ state also gives significant fission yield within PPP model due to mixing of $2^{1} A$ state in the eigenstates. For higher donor-acceptor strengths, the singlet state derived from the $2^{1} A$ state loses its two-triplet character and the fission yield goes down significantly. We have also found that fission yield depends on stacking geometry. In $\mathrm{V}$ stacking where the intermolecular transfer integral $t_{\perp}>0$, the singlet fission yield in the PPP model decreases with increasing chain length while when $t_{\perp}<0$ as in $\mathrm{H}$ stacking, there is an increase in fission yield. We expect SF to occur from the lowest excited singlet state, as fast internal conversions lead to this state independent of the initial excited state reached by photoexcitation. Hence, in systems where the lowest singlet excited state is the state with large $2^{1} A$ character, we expect significant SF yield. However, if the lowest excited singlet state has largely $1^{1} B$ character, the SF yield will be negligible. Thus, we can see that systems which are fluorescent will not give large SF yields. Therefore, systems which are good for light emission will not be good candidates for improving photovoltaic efficiency through SF.

\section{ACKNOWLEDGMENTS}

S.R. is thankful to the Department of Science and Technology, India for financial support through various grants. S.P. acknowledges CSIR India for a senior research fellowship. S.P. also thanks Prof. Diptiman Sen for financial support through his J. C. Bose fellowship.
${ }^{1}$ S. Singh, W. J. Jones, W. Siebrand, B. P. Stoicheff, and W. G. Schneider, J. Chem. Phys. 42, 330 (1965); C. E. Swenberg and W. T. Stacy, Chem. Phys. Lett. 2, 327 (1968); R. E. Merrifield, P. Avakian, and R. P. Groff, ibid. 3, 386 (1969); R. P. Groff, P. Avakian, and R. E. Merrifield, Phys. Rev. B 1, 815 (1970).

2 R. E. Merrifield, J. Chem. Phys. 48, 4318 (1968); R. C. Johnson and R. E. Merrifield, Phys. Rev. B 1, 896 (1970).

3 M. B. Smith and J. Michl, Chem. Rev. 110, 6891 (2010).

4 J. C. Johnson, A. J. Nozik, and J. Michl, Acc. Chem. Res. 46, 1290 (2013).

${ }^{5}$ M. B. Smith and J. Michl, Annu. Rev. Phys. Chem. 64, 361 (2013).

${ }^{6}$ W.-L. Chan, M. Ligges, A. Jailaubekov, L. Kaake, L. Miaja-Avila, and X.-Y. Zhu, Science 334, 1541 (2011).

7 H. L. Stern, A. J. Musser, S. Gelinas, P. Parkinson, L. M. Herz, M. J. Bruzek, J. Anthony, R. H. Friend, and B. J. Walker, Proc. Natl. Acad. Sci. U.S.A. 112, 7656 (2015).

8 I. Paci, J. C. Johnson, X. Chen, G. Rana, D. Popović, D. E. David, A. J. Nozik, M. A. Ratner, and J. Michl, J. Am. Chem. Soc. 128, 16546 (2006).

${ }^{9}$ E. C. Greyson, B. R. Stepp, X. Chen, A. F. Schwerin, I. Paci, M. B. Smith, A. Akdag, J. C. Johnson, A. J. Nozik, J. Michl, and M. A. Ratner, J. Phys. Chem. B 114, 14223 (2010).

10 T. Minami and M. Nakano, J. Phys. Chem. Lett. 3, 145 (2012).

11 T. Minami, S. Ito, and M. Nakano, J. Phys. Chem. Lett. 3, 2719 (2012)

12 T. Minami, S. Ito, and M. Nakano, J. Phys. Chem. Lett. 4, 2133 (2013).

13 P. M. Zimmerman, Z. Zhang, and C. B. Musgrave, Nat. Chem. 2, 648 (2010).

14 P. M. Zimmerman, F. Bell, D. Casanova, and M. HeadGordon, J. Am. Chem. Soc. 133, 19944 (2011).

15 P. M. Zimmerman, C. B. Musgrave, and M. Head-Gordon,
Acc. Chem. Res. 46, 1339 (2013).

16 T. Zeng, N. Ananth, and R. Hoffmann, J. Am. Chem. Soc. 136, 12638 (2014).

17 T. Zeng, R. Hoffmann, and N. Ananth, J. Am. Chem. Soc. 136, 5755 (2014).

18 E. G. Fuemmeler, S. N. Sanders, A. B. Pun, E. Kumarasamy, T. Zeng, K. Miyata, M. L. Steigerwald, X.-Y. Zhu, M. Y. Sfeir, L. M. Campos, and N. Ananth, ACS Cent. Sci. 2, 316 (2016).

19 R. W. A. Havenith, H. D. de Gier, and R. Broer, Mol. Phys. 110, 2445 (2012).

20 S. M. Parker, T. Seideman, M. A. Ratner, and T. Shiozaki, J. Phys. Chem. C 118, 12700 (2014).

${ }^{21}$ K. Aryanpour, A. Shukla, and S. Mazumdar, J. Phys. Chem. C 119, 6966 (2015).

22 C. C. Gradinaru, J. T. M. Kennis, E. Papagiannakis, I. H. M. van Stokkum, R. J. Cogdell, G. R. Fleming, R. A. Niederman, and R. van Grondelle, Proc. Natl. Acad. Sci. U.S.A. 98, 2364 (2001).

${ }^{23}$ E. Papagiannakis, J. T. M. Kennis, I. H. M. van Stokkum, R. J. Cogdell, and R. van Grondelle, Proc. Natl. Acad. Sci. U.S.A. 99, 6017 (2002).

${ }^{24}$ C. Wang and M. J. Tauber, J. Am. Chem. Soc. 132, 13988 (2010).

25 C. Wang, M. Angelella, C.-H. Kuo, and M. J. Tauber, Proc. SPIE 8459, 845905 (2012).

26 R. J. Dillon, G. B. Piland, and C. J. Bardeen, J. Am. Chem. Soc. 135, 17278 (2013).

27 A. J. Musser, M. Al-Hashimi, M. Maiuri, D. Brida, M. Heeney, G. Cerullo, R. H. Friend, and J. Clark, J. Am. Chem. Soc. 135, 12747 (2013).

28 A. J. Musser, M. Maiuri, D. Brida, G. Cerullo, R. H. Friend, and J. Clark, J. Am. Chem. Soc. 137, 5130 (2015).

29 X. Feng, A. V. Luzanov, and A. I. Krylov, J. Phys. Chem. Lett. 4, 3845 (2013).

30 N. Renaud, P. A. Sherratt, and M. A. Ratner, J. Phys. 
Chem. Lett. 4, 1065 (2013).

31 S. W. Eaton, L. E. Shoer, S. D. Karlen, S. M. Dyar, E. A. Margulies, B. S. Veldkamp, C. Ramanan, D. A. Hartzler, S. Savikhin, T. J. Marks, and M. R. Wasielewski, J. Am. Chem. Soc. 135, 1470114712 (2013).

32 L. Wang, Y. Olivier, O. V. Prezhdo, and D. Beljonne, J. Phys. Chem. Lett. 5, 3345 (2014).

33 N. Renaud and F. C. Grozema, J. Phys. Chem. Lett. 6, 360 (2015).

34 B. J. Walker, A. J. Musser, D. Beljonne, and R. H. Friend, Nat. Chem. 5, 1019 (2013).

35 J. Zirzlmeier, D. Lehnherr, P. B. Coto, E. T. Chernick, R. Casillas, B. S. Basel, M. Thoss, R. R. Tykwinskid, and D. M. Guldi, Proc. Natl. Acad. Sci. U.S.A. 112, 5325 (2013).

36 S. N. Sanders, E. Kumarasamy, A. B. Pun, M. T. Trinh, B. Choi, J. Xia, E. J. Taffet, J. Z. Low, J. R. Miller, X. Roy, X.-Y. Zhu, M. L. Steigerwald, M. Y. Sfeir, and L. M. Campos, J. Am. Chem. Soc. 137, 8965 (2015).

37 B. S. Hudson and B. E. Kohler, Chem. Phys. Lett. 14, 299 (1972); K. Schulten and M. Karplus, ibid. 14, 305 (1972).

38 P. Tavan and K. Schulten, Phys. Rev. B 36, 4337 (1987).

39 E. C. Greyson, J. Vura-Weis, J. Michl, and M. A. Ratner, J. Phys. Chem. B 114, 14168 (2010).

40 Y. Chen, L. Shen, and X. Li, J. Phys. Chem. A 118, 5700 (2014).

41 E. Busby, J. Xia, Q. Wu, J. Z. Low, R. Song, J. R. Miller, X.-Y. Zhu, L. M. Campos, and M. Y. Sfeir, Nat. Mater. 14, $426(2015)$

${ }^{42}$ K. Aryanpour, T. Dutta, U. N. V. Huynh, Z. V. Vardeny, and S. Mazumdar, Phys. Rev. Lett. 115, 267401 (2015).

43 Y. Kasai, Y. Tamai, H. Ohkita, H. Benten, and S. Ito, J. Am. Chem. Soc. 137, 15980 (2015).

44 A. M. Müller, Y. S. Avlasevich, K. Müllen, and C. J. Bardeen, Chem. Phys. Lett. 421, 518 (2006).

45 A. M. Müller, Y. S. Avlasevich, W. W. Schoeller, K. Müllen, and C. J. Bardeen, J. Am. Chem. Soc. 129, 14240 (2007).

${ }^{46}$ P. J. Vallett, J. L. Snyder, and N. H. Damrauer, J. Phys. Chem. A 117, 10824 (2013).

47 N. V. Korovina, S. Das, Z. Nett, X. Feng, J. Joy, R. Haiges, A. I. Krylov, S. E. Bradforth, and M. E. Thompson, J. Am. Chem. Soc. 138, 617 (2016).

48 X. Feng, D. Casanova, and A. I. Krylov, J. Phys. Chem. C 120, 19070 (2016).

49 J. D. Cook, T. J. Carey, and N. H. Damrauer, J. Phys. Chem. A 120, 4473 (2016).

50 S. Lukman, A. J. Musser, K. Chen, S. Athanasopoulos, C. K. Yong, Z. Zeng, Q. Ye, C. Chi, J. M. Hodgkiss, J. Wu, R. H. Friend, , and N. C. Greenham, Adv. Funct. Mater. 25, 5452 (2015).

51 S. N. Sanders, E. Kumarasamy, A. B. Pun, M. L. Steigerwald, M. Y. Sfeir, and L. M. Campos, Angew. Chem. 128, 3434 (2016).

52 S. N. Sanders, E. Kumarasamy, A. B. Pun, K. Appavoo, M. L. Steigerwald, L. M. Campos, and M. Y. Sfeir, J. Am. Chem. Soc. 138, 7289 (2016).

53 T. Sakuma, H. Sakai, Y. Araki, T. Mori, T. Wada, N. V. Tkachenko, and T. Hasobe, J. Phys. Chem. A 120, 1867 (2016).

54 S. Ito, T. Nagami, and M. Nakano, J. Phys. Chem. A 120, 6236 (2016).

55 J. Zirzlmeier, R. Casillas, S. R. Reddy, P. B. Coto, D. Lehn- herr, E. T. Chernick, I. Papadopoulos, M. Thoss, R. R. Tykwinski, and D. M. Guldi, Nanoscale 8, 10113 (2016).

56 S. Lukman, K. Chen, J. M. Hodgkiss, D. H. P. Turban, N. D. M. Hine, S. Dong, J. Wu, N. C. Greenham, and A. J. Musser, Nat. Commun. 7, 13622 (2016).

57 S. N. Sanders, E. Kumarasamy, A. B. Pun, M. L. Steigerwald, M. Y. Sfeir, and L. M. Campos, Chem 1, 505 (2016).

58 J. C. Johnson, A. Akdag, M. Zamadar, X. Chen, A. F. Schwerin, I. Paci, M. B. Smith, Z. Havlas, J. R. Miller, M. A. Ratner, A. J. Nozik, and J. Michl, J. Phys. Chem. B 117, 4680 (2013).

59 E. A. Margulies, C. E. Miller, Y. Wu, L. Ma, G. C. Schatz, R. M. Young, and M. R. Wasielewski, Nat. Chem. 8, 1120 (2016).

60 O. Varnavski, N. Abeyasinghe, J. Aragó, J. J. SerranoPérez, E. Ortí, J. T. López Navarrete, K. Takimiya, D. Casanova, J. Casado, and T. Goodson, III, J. Phys. Chem. Lett. 6, 1375 (2015).

61 A. D. Chien, A. R. Molina, N. Abeyasinghe, O. P. Varnavski, T. Goodson, III, and P. M. Zimmerman, J. Phys. Chem. C 119, 28258 (2015).

62 R. Pariser and R. G. Parr, J. Chem. Phys. 21, 466 (1953). 63 J. A. Pople, Trans. Faraday Soc. 49, 1375 (1953).

${ }^{64}$ K. Ohno, Theor. Chim. Acta 2, 219 (1964); G. Klopman, J. Am. Chem. Soc. 86, 4550 (1964).

65 A. Race, W. Barford, and R. J. Bursill, Phys. Rev. B 64, 035208 (2001); 67, 245202 (2003).

66 Z. G. Soos and S. Ramasesha, Phys. Rev. B 29, 5410 (1984).

67 S. Ramasesha and Z. G. Soos, Int. J. Quan. Chem. 25, 1003 (1984).

68 D. K. Campbell, J. T. Gammel, and E. Y. Loh, Jr, Phys. Rev. B 42, 475 (1990); K. Tandon, S. Ramasesha, and S. Mazumdar, 67, 045109 (2003).

69 M. Wolfsberg and L. Helmholz, J. Chem. Phys. 20, 837 (1952); R. Hoffmann and W. N. Lipscomb, 36, 2179 (1962).

70 See Supplemental Material at [] for properties of low-lying singlet states of butadiene and hexatriene dimers, projection of $1^{1} B \otimes 1^{1} A$ and $T_{1} \otimes T_{1}$ with eigenstates of the dimer system within Hückel and Hubbard model and time evolution profiles of unsubstituted and substituted butadiene and hexatriene dimers.

71 T. Iitaka, Phys. Rev. E 49, 4684 (1994).

72 T. Dutta and S. Ramasesha, Computing Letters 3, 457 (2007).

73 S. C. Chapra and R. P. Canale, Numerical Methods for Engineers, 6th ed. (McGraw Hill Education (India) Pvt. Ltd., New Delhi, 2012).

74 J. Crank and P. Nicolson, Mathematical Proceedings of the Cambridge Philosophical Society 43, 50 (1947).

75 I. I. Rabi, Phys. Rev. 51, 652 (1937).

76 P. Tavan and K. Schulten, J. Chem. Phys. 70, 5407 (1979); 70, 5414 (1979); 85, 6602 (1986).

77 Z. G. Soos, S. Ramasesha, and D. S. Galvão, Phys. Rev. Lett. 71, 1609 (1993)

78 D. Baeriswyl, D. K. Campbell, and S. Mazumdar, Conjugated Conducting Polymers, edited by H. Kiess, Springer Series in Solid-State Sciences, Vol. 102 (Springer, Berlin, 1992).

79 Z. Shuai, J. L. Brédas, S. K. Pati, and S. Ramasesha, Phys. Rev. B 56, 9298 (1997).

80 J. Ren, Q. Peng, X. Zhang, Y. Yi, and Z. Shuai, J. Phys. Chem. Lett. 8, 2175 (2017). 